\section{"Social Physiology" for Psychiatric Semiology: How TTOM can initiate an interactive turn for Computational Psychiatry?}

\begin{abstract}
Guillaume Dumas ${ }^{a, b,{ }^{*}}$, Tudi Gozéc,d, and
Jean-Arthur Micoulaud-Franchie,f

a Human Genetics and Cognitive Functions, Institut Pasteur, UMR3571 CNRS, Université de Paris, Paris, France; ${ }^{b}$ Human Brain and Behavior Laboratory, Center for Complex Systems and Brain Sciences, Florida Atlantic University, Boca Raton, FL, USA; ' Department of Psychiatry, Psychotherapies, Art-therapy, Toulouse University Hospital, France; ${ }^{d}$ Equipe de Recherche sur les Rationalités Philosophiques et les Savoirs EA3051, Université de Toulouse - Jean Jaurès, France; e Services d'explorations fonctionnelles du système nerveux, Clinique du sommeil, CHU de Bordeaux, Place Amélie Raba-Leon, 33076 Bordeaux, France; ${ }^{f}$ USR CNRS 3413 SANPSY, CHU Pellegrin, Université de Bordeaux, France. guillaume.dumas@pasteur.fr
\end{abstract}

\section{Abstract: Thinking Through Other Minds (TTOM) encompasses new dimensions in computational psychiatry: social interaction and mutual sense-making. It questions the nature of psychiatric manifestations (semiology) in the light of recent data on social interaction in neuroscience. We propose the concept of "social physiology" in response to the call by the conceivers of TTOM for the renewal of computational psychiatry.}

Psychiatric semiology, i.e. the science of clinical manifestations, considers that both symptoms and signs are "units of analysis". These units are actionable psychopathological features that are essential in practice for making the diagnosis and prognosis that underpin clinical decision-making (Micoulaud-Franchi et al., 2018). Psychiatric semiology is therefore very important. We would like to comment on how the concept of TTOM and the associated computational psychiatry model proposed by the authors not only questions the mechanisms underlying psychiatric manifestations (see p63-64, Veissière, Constant, Ramstead, Friston, \& Kirmayer, 2019) but also the ways in which these manifestations are expressed by patients and captured by psychiatrists. Indeed, we need a computational model that questions how clinical manifestations are expressed and captured. In the field of transcultural (Kirmayer \& Crafa, 2014) and phenomenological psychiatry (Nordgaard, Sass, \& Parnas, 2013), it is widely considered that semiology is partially based on social and cultural construction (i.e. history of medicine, consensus of experts, folk psychology, etc.; Kirmayer \& Ramstead, 2017). Moreover, with a hand outstretched to medicine, psychiatry considers that symptoms and signs are in some way linked to physiologic disturbances in the brain, as investigated by neuroscience (JeanArthur Micoulaud-Franchi, Dumas, Quiles, \& Vion-Dury, 2016). Since Jaspers' work, clinical manifestations have been taken to reflect both physiologic disturbances and patients' attitudes toward them (Stanghellini, Bolton, \& Fulford, 2013). In this view, clinical manifestations are not just related to an underlying physiologic cause but also to cognitive-interpretive and interpersonal processes that are at play during the constitution of symptoms and signs (Kirmayer \& Sartorius, 2007; Kirmayer \& Ramstead, 2017). Although theories have been proposed to account for these two dimensions central to psychiatric semiology (Berrios, 1996; Borsboom, Cramer, \& Kalis, 2018; Kirmayer \&
Ramstead, 2017), none has been formalized with a computational model. While TTOM is a welcome addition that could help in formalising clinical manifestations expressed and captured at the cultural/social level, we think that it should also take the recent advances in the neuroscience of social interaction into account.

In the last decade, social neuroscience has indeed become interactive in acknowledging the impact of interpersonal social dynamics on intra-personal neurobehavioral dynamics (Hari \& Kujala, 2009; Redcay \& Schilbach, 2019). The second-person perspective (Schilbach et al., 2013) has already led to the development of a "second-person neuropsychiatry" that considers psychiatric disorders as "disturbances of social cognition" (Schilbach, 2016). Complementary to this perspective, two-body neuroscience and two-person physiology (Bolis \& Schilbach, 2018; Dumas, 2011) follow the call for radical embodiment in cognitive science (Thompson \& Varela, 2001) and emphasize the constitutive role of interpersonal dynamics in individual cognition. Hyperscanning, i.e. the simultaneous brain recording of several people (Montague et al., 2002), has demonstrated how non-verbal interaction through sensorimotor loops modulates individuals respective internal neurophysiological dynamics and how interpersonal dynamics are measurable at the electrophysiological level through inter-brain synchronizations (Dumas, Nadel, Soussignan, Martinerie, \& Garnero, 2010). While it does not negate the existence of higher-order representations (e.g. language, cultural habitus; Shea et al., 2014), it supports the development of a "social physiology" that continuously integrates sensorimotor and representational levels of analysis. Interestingly, TTOM is already trying to resolve the difference between the individual and the inter-individual, and the authors present embodied interaction as always being culturally coded by implicit cultural learning. However, in our opinion, there is also a more basic non-culturally coded and non-representational layer of interaction that is directly rooted in early developmental processes.

This layer of interaction is particularly relevant for studying neurodevelopmental disorders such as autism and schizophrenia. In autism, multi-scale approaches have already attempted to solve this social paradox (Bolis, Balsters, Wenderoth, Becchio, \& Schilbach, 2017; Dumas, Kelso, \& Nadel, 2014). In schizophrenia, the phenomenological approach has been used to account for impairment in the ability to learn the implicit social senses and use them in non-verbal communication (Fuchs, 2015). This involves a nonverbal and pre-individual layer of relation (Lavelle, Healey, \& McCabe, 2014) that is closely linked to minimal-self disorder as an alteration of the first-person perspective (Parnas \& Zandersen, 2018). This alteration raises the question how symptoms are expressed by patients and captured by psychiatrists. This issue has been analysed within the framework of the classical concept of "Praecox Feeling". This "feeling" of bizarreness in interaction can be considered as a crucial determinant of medical decision-making in psychiatry (Cermolacce, Sass, \& Parnas, 2010; Gozé et al., 2018), because it is directly rooted in minimal-self disorders (Parnas, 2011; Sass, Borda, Madeira, Pienkos, \& Nelson, 2018). These well-documented first-person accounts suggest that patients are affected at a more basic level than TTOM. Hence, this suggests the existence of a sub-layer of TTOM itself which could be impaired, so the mechanisms underlying the involvement of 
TTOM require their own model. To meet this objective, social physiology calls for a computational model under (nonrepresentational) and beyond (implicit or explicit social representations) the individual. While Bayesian statistics can virtually integrate these dimensions (Friston \& Frith, 2015), they need to be captured by generative models that are based on other types of computational formalism (Friston, Redish, \& Gordon, 2017; Montague, Dolan, Friston, \& Dayan, 2011), especially biophysically-based neural circuit models (Wang \& Krystal, 2014). In our view, while TTOM provides a good matrix to model psychiatric semiology and its relation both to physiology and to social interaction, it also requires the development of complementary computational models to account for physiological brain mechanisms and non-representational interpersonal dynamics (Dumas, Chavez, Nadel, \& Martinerie, 2012). The goal is to encompass the neurophysiological level and not separate the "implementation" from the "computational" in Marr's sense (Marr, 1982). Such TTOM combined with social physiology, including all three of Marr's levels (computational, algorithms, implementation), could offer great perspectives for our understanding of how psychiatric manifestations are expressed and captured. This could help computational psychiatry to structure the classification of mental disorders, even including the more tacit mechanisms of intuition in clinical decision-making.

\section{REFERENCES}

Berrios, G. E. (1996). The history of mental symptoms: Descriptive psychopathology since the nineteenth century. Cambridge University Press.

Bolis, D., Balsters, J., Wenderoth, N., Becchio, C., \& Schilbach, L. (2017). Beyond Autism: Introducing the Dialectical Misattunement Hypothesis and a Bayesian Account of Intersubjectivity. Psychopathology, 50(6), 355-372. https://doi.org/10.1159/000484353

Bolis, D., \& Schilbach, L. (2018). 'I Interact Therefore I Am': The Self as a Historical Product of Dialectical Attunement. Topoi. https://doi.org/10.1007/s11245-018-9574-0

Borsboom, D., Cramer, A., \& Kalis, A. (2018). Brain disorders? Not really... Why network structures block reductionism in psychopathology research. The Behavioral and Brain Sciences, 1-54. https://doi.org/10.1017/S0140525X17002266

Cermolacce, M., Sass, L., \& Parnas, J. (2010). What is Bizarre in Bizarre Delusions? A Critical Review. Schizophrenia Bulletin, 36(4), 667679. https://doi.org/10.1093/schbul/sbq001

Dumas, G. (2011). Towards a two-body neuroscience. Communicative \& Integrative Biology, 4(3), 349-352. https://doi.org/10.4161/cib.4.3.15110

Dumas, G., Kelso, J. A. S., \& Nadel, J. (2014). Tackling the social cognition paradox through multi-scale approaches. Cognitive Science, 5, 882. https://doi.org/10.3389/fpsyg.2014.00882

Dumas, G., Nadel, J., Soussignan, R., Martinerie, J., \& Garnero, L. (2010). Inter-brain synchronization during social interaction. PLOS ONE, 5(8), e12166. https://doi.org/10.1371/journal.pone.0012166

Friston, K. J., Redish, A. D., \& Gordon, J. A. (2017). Computational Nosology and Precision Psychiatry. Computational Psychiatry, 1-22. https://doi.org/10.1162/CPSY_a_00001

Gozé, T., Moskalewicz, M., Schwartz, M. A., Naudin, J., MicoulaudFranchi, J.-A., \& Cermolacce, M. (2018). Reassessing "Praecox Feeling" in Diagnostic Decision Making in Schizophrenia: A Critical Review. Schizophrenia Bulletin. https://doi.org/10.1093/schbul/sby172

Hari, R., \& Kujala, M. V. (2009). Brain basis of human social interaction: From concepts to brain imaging. Physiological Reviews, 89(2), 453479. https://doi.org/10.1152/physrev.00041.2007

Kirmayer, L. J., \& Crafa, D. (2014). What kind of science for psychiatry? Frontiers in Human Neuroscience, 8. https://doi.org/10.3389/fnhum.2014.00435
Kirmayer, L. J., \& Sartorius, N. (2007). Cultural Models and Somatic Syndromes. Psychosomatic Medicine, 69(9), 832. https://doi.org/10.1097/PSY.0b013e31815b002c

Kirmayer, L., \& Ramstead, M. (2017). Embodiment and enactment in cultural psychiatry. In Embodiment, Enaction, and Culture: Investigating the Constitution of the Shared World (pp. 397-422).

Lavelle, M., Healey, P. G. T., \& McCabe, R. (2014). Nonverbal behavior during face-to-face social interaction in schizophrenia: A review. The Journal of Nervous and Mental Disease, 202(1), 47-54. https://doi.org/10.1097/NMD.0000000000000031

Micoulaud-Franchi, J.-A., Quiles, C., Batail, J.-M., Lancon, C., Masson, M., Dumas, G., \& Cermolacce, M. (2018). Making psychiatric semiology great again: A semiologic, not nosologic challenge. L'Encephale, 44(4), 343-353. https://doi.org/10.1016/j.encep.2018.01.007

Micoulaud-Franchi, Jean-Arthur, Dumas, G., Quiles, C., \& Vion-Dury, J. (2016). From clinic to the "foul and exciting field of life": A psychiatric point of view on clinical physiology. Annales Médicopsychologiques, revue psychiatrique. https://doi.org/10.1016/j.amp.2016.11.004

Montague, P. R., Berns, G. S., Cohen, J. D., McClure, S. M., Pagnoni, G., Dhamala, M., ... Fisher, R. E. (2002). Hyperscanning: Simultaneous fMRI during Linked Social Interactions. NeuroImage, 16(4), 1159 1164. https://doi.org/10.1006/nimg.2002.1150

Montague, P. R., Dolan, R. J., Friston, K. J., \& Dayan, P. (2011). Computational psychiatry. Trends in Cognitive Sciences, 1-9. https://doi.org/10.1016/j.tics.2011.11.018

Nordgaard, J., Sass, L. A., \& Parnas, J. (2013). The psychiatric interview: Validity, structure, and subjectivity. European Archives of Psychiatry and Clinical Neuroscience, 263(4), 353-364. https://doi.org/10.1007/s00406-012-0366-z

Parnas, J. (2011). A disappearing heritage: The clinical core of schizophrenia. Schizophrenia Bulletin, 376), 1121-1130.

Parnas, J., \& Zandersen, M. (2018). Self and schizophrenia: Current status and diagnostic implications. World Psychiatry, 1ス2), 220221. https://doi.org/10.1002/wps. 20528

Redcay, E., \& Schilbach, L. (2019). Using second-person neuroscience to elucidate the mechanisms of social interaction. Nature Reviews Neuroscience. https://doi.org/10.1038/s41583-019-0179-4

Sass, L., Borda, J. P., Madeira, L., Pienkos, E., \& Nelson, B. (2018). Varieties of self disorder: A bio-pheno-social model of schizophrenia. Schizophrenia Bulletin, 44(4), 720-727.

Schilbach, L. (2016). Towards a second-person neuropsychiatry. Philosophical Transactions of the Royal Society B: Biological Sciences, 371(1686). https://doi.org/10.1098/rstb.2015.0081

Schilbach, L., Timmermans, B., Reddy, V., Costall, A., Bente, G., Schlicht, T., \& Vogeley, K. (2013). Toward a second-person neuroscience. Behavioral and Brain Sciences, 36(04), 393-414. https://doi.org/10.1017/S0140525X12000660

Shea, N., Boldt, A., Bang, D., Yeung, N., Heyes, C., \& Frith, C. D. (2014). Supra-personal cognitive control and metacognition. Trends in Cognitive Sciences, 18(4), 186-193. https://doi.org/10.1016/j.tics.2014.01.006

Stanghellini, G., Bolton, D., \& Fulford, W. K. M. (2013). Person-Centered Psychopathology of Schizophrenia: Building on Karl Jaspers' Understanding of Patient's Attitude Toward His Illness. Schizophrenia Bulletin, 39(2), 287-294. https://doi.org/10.1093/schbul/sbs154

Thompson, E., \& Varela, F. J. (2001). Radical embodiment: Neural dynamics and consciousness. Trends in Cognitive Sciences, 5(10), 418-425.

Veissière, S. P. L., Constant, A., Ramstead, M. J. D., Friston, K. J., \& Kirmayer, L. J. (2019). Thinking Through Other Minds: A Variational Approach to Cognition and Culture. Behavioral and Brain Sciences, 1-97. https://doi.org/10.1017/S0140525X19001213

Wang, X.-J., \& Krystal, J. H. (2014). Computational Psychiatry. Neuron, 84(3), 638-654. https://doi.org/10.1016/j.neuron.2014.10.018 